Wien klin Mag 2021 $24: 246-251$ https://doi.org/10.1007/s00740-021-00414-z Online publiziert: 21. Oktober 2021 (c) Springer-Verlag GmbH Austria, ein Teil von Springer Nature 2021

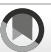

\section{Dennis A. Eichenauer' - Gunnar Lachmann² - Paul La Rosée}

' Klinik I für Innere Medizin, Zentrum für Integrierte Onkologie Aachen Bonn Köln Düsseldorf, Uniklinik Köln, Köln, Deutschland

${ }^{2}$ Klinik für Anästhesiologie mit Schwerpunkt Operative Intensivmedizin, Charité - Universitätsmedizin Berlin, Berlin, Deutschland

${ }^{3}$ Klinik für Innere Medizin II, Schwarzwald-Baar-Klinikum, Villingen-Schwenningen, Deutschland

\title{
Die hämophagozytische Lymphohistiozytose bei kritisch kranken Patienten
}

Die hämophagozytische Lymphohistiozytose (HLH) ist ein potenziell lebensbedrohliches Hyperinflammationssyndrom. Unterschieden wird die primäre, genetisch bedingte $\mathrm{HLH}$, die sich zumeist im Kindesalter manifestiert, von der sekundären, erworbenen HLH, die Patienten aller Altersgruppen betreffen kann [16]. Bei der sekundären HLH stellen Infektionen, Malignome und $\mathrm{Au}$ toimmunerkrankungen häufige Trigger dar [2]. Nicht immer kann jedoch ein Trigger identifiziert werden. Aufgrund des unspezifischen klinischen Erscheinungsbilds, das von einer Sepsis häufig kaum zu unterscheiden ist, wird die Diagnose HLH in vielen Fällen nicht oder erst spät gestellt, was zu einem verzögerten Behandlungsbeginn und dadurch zu einer Verschlechterung der Prognose führt. Dies gilt in besonderem Maß für kritisch kranke Patienten auf der Intensivstation, bei denen Sterberaten zwischen $50 \%$ und $80 \%$ beschrieben sind $[12,18]$.

\section{Pathophysiologie und klinisches Bild}

Je nach auslösendem Trigger wird zwischen der HLH (z. B. bei Infektionen und Malignomen) und dem Makropha-

Dieser Beitrag wurde in der Zeitschrift Medizinische Klinik - Intensivmedizin und Notfallmedizin $2 \cdot 2021$; 116:129-134. https://doi.org/10. 1007/s00063-021-00781-9 erstveröffentlicht. Zweitpublikation mit freundlicher Genehmigung der Autoren. genaktivierungssyndrom (MAS-HLH) unterschieden. Das MAS-HLH stellt die Endstrecke der HLH bei rheumatologischen Grunderkrankungen dar. Pathophysiologisch liegen den Subtypen der HLH komplexe Dysregulationen von T-Zell-Antwort, Makrophagenaktivierung und Inflammasom zugrunde. Es kommt zu einer überschießenden Entzündungsreaktion mit Zytokinsturm und ausgeprägter Hyperferritinämie. Die namensgebende Phagozytose hämatopoetischer Zellen durch Makrophagen kann zusammen mit dem häufig aberrant proliferationsaktiven zytotoxischen T-Zell-Kompartiment zu einem Multiorganversagen führen [3].

\section{》) Protrahiert hohes Fieber unklarer Genese stellt das führende Symptom der HLH dar}

Klinisch stellt protrahiert hohes Fieber, das unzureichend auf eine empirische antiinfektive Therapie anspricht, das führende Symptom der HLH dar. Liegen zusätzlich eine Vergrößerung von Milz und/oder Leber sowie eine Bi- oder Panzytopenie vor, sollte eine $\mathrm{HLH}$ in Betracht gezogen und entsprechende Diagnostik durchgeführt werden. Die Höhe des C-reaktiven Proteins (CRP) ist bei der HLH in Relation zu den klinischen Inflammationszeichen zumeist auffällig niedrig. Beim MAS-HLH dagegen zeigen sich zunächst durchaus eine Leukozytose sowie eine deutliche Erhöhung von CRP und Fibrinogen, bevor bei weiterem Fortschreiten schrittweise die HLH-Kriterien erfüllt werden. Weitere im Rahmen einer HLH fakultativ auftretende Symptome und laborchemische Veränderungen beinhalten neurologische Auffälligkeiten, vergrößerte Lymphknoten, eine Erhöhung der Transaminasen und eine eingeschränkte Syntheseleistung der Leber, die sich insbesondere durch Gerinnungsstörungen manifestiert.

\section{Diagnosestellung}

Die Diagnose HLH wird mithilfe der HLH-2004-Kriterien gestellt (• Tab. 1; - Abb. 1). Neben der Symptomentrias Fieber, Splenomegalie und Zytopenie fließen hier die Parameter Ferritinämie, Triglyzeridämie und/oder Hypofibrinogenämie, erhöhter löslicher Interleukin(IL)-2-Rezeptor, verminderte Natural-Killer(NK)-Zell-Aktivität und Nachweis einer Hämophagozytose in Knochenmark, Lymphknoten oder Liquor ein. Bei Vorliegen von wenigstens $5 \mathrm{der}$ 8 Kriterien kann die Diagnose HLH gestellt werden [10]. Sensitivität und Spezifität der HLH-Kriterien wurden hauptsächlich bei pädiatrischen Patienten untersucht. Es gibt jedoch auch Untersuchungen bei kritisch kranken erwachsenen Patienten, die eine Sensitivität zwischen $70 \%$ und $95 \%$ sowie eine Spezifität zwischen $90 \%$ und 93,6\% zeigten [13, 22].

Neben den HLH-2004-Kriterien wurde der HScore entwickelt und validiert, 
Tab. 1 HLH-2004 Diagnosekriterien

( 5 von 8 müssen für Diagnose einer HLH vorliegen)

1 Fieber

2 Splenomegalie

3 Zytopenie ( $\geq 2$ von 3 Linien im peripheren Blut):

Hämoglobin $<9 \mathrm{~g} / \mathrm{d}$

Thrombozyten $<100 \times 10^{9} / \mathrm{I}$

Neutrophile $<1,0 \times 10^{9} /$ I

4 Hypertriglyzeridämie und/oder Hypofibrinogenämie

Nüchterntriglyzeride $\geq 265 \mathrm{mg} / \mathrm{dl}$

Fibrinogen $\leq 1,5 \mathrm{~g} / \mathrm{l}$

5 Hämophagozytose in Knochenmark, Milz oder Lymphknoten

6 Niedrige Natural-Killer(NK)-Zell-Aktivität

7 Ferritin $\geq 500 \mu \mathrm{g} / \mathrm{l}$

8 Löslicher Interleukin-2-Rezeptor $\geq 2400 \mathrm{U} / \mathrm{ml}$

Bemerkungen

(1) Fehlende Hämophagozytose schließt das Vorliegen einer HLH nicht aus

(2) Diagnosestützend sind zerebromeningeale Symptome, Liquorpleozytose und Eiweißerhöhung im Liquor, Lymphknotenvergrößerung, Ikterus, Ödeme, Hautexanthem, Transaminasenerhöhung, Hypalbuminämie, Hyponatriämie sowie eine bioptisch gesicherte chronisch persistierende Hepatitis

HLH Hämophagozytische Lymphohistiozytose, $C D$ Differenzierungscluster

mit dessen Hilfe die Wahrscheinlichkeit für das Vorliegen einer HLH berechnet werden kann. Er besteht aus 9 Parametern, die in großen Teilen mit den HLH2004-Kriterien übereinstimmen. Für den HScore werden die einzelnen Faktoren jedoch nicht nur qualitativ, sondern auch quantitativ berücksichtigt $[4,5]$. Wird im HScore ein Cut-off-Wert von 168 gesetzt, konnte für kritisch kranke erwachsene Patienten eine Sensitivität von $100 \%$ und eine Spezifität von 94,1\% im Hinblick auf die Diagnose einer HLH gezeigt werden [13]. Auf der Intensivstation kommt zudem dem Ferritinwert als Screening- und Verlaufsparameter besondere Bedeutung zu $[15,19]$. So fanden sich im Rahmen einer Analyse, in die 2623 kritisch kranke Patienten mit einer Hyperferritinämie größer $500 \mu \mathrm{g} / \mathrm{l}$ eingeschlossen wurden, bei Patienten mit HLH $(n=40)$ signifikant höhere maximale Ferritinwerte als bei Patienten mit Sepsis, septischem Schock oder ande- ren Diagnosen. Wird der Cut-off-Wert bei einem maximalen Ferritinwert von $9083 \mu \mathrm{g} / \mathrm{l}$ gesetzt, liegen Sensitivität und Spezifität im Hinblick auf das Vorliegen einer HLH bei 92,5\% beziehungsweise 91,9\% [19].

An die Stellung der Diagnose HLH anhand der genannten Kriterien schließt sich die Suche nach einem möglichen auslösenden Trigger an. Gemäß einer systematischen Übersichtsarbeit, in die 661 kritisch kranke erwachsene HLH-Patienten eingeschlossen wurden, stellen Infektionen (50\%), Malignome (28\%) und Autoimmunerkrankungen (12\%) auf der Intensivstation die häufigsten auslösenden Faktoren dar [14].

\section{Therapie und Verlauf}

Neben der supportiven intensivmedizinischen Versorgung, die eine invasive Beatmung, die Gabe von Vasopressoren, die Nierenersatztherapie und weitere Therapieverfahren beinhalten kann, benötigen HLH-Patienten eine immunsuppressive Behandlung zur Unterdrückung der Hyperinflammation. Der Grundpfeiler der Therapie besteht im Einsatz von hoch dosierten Kortikosteroiden (• Abb. 1). Die darüber hinausgehende Behandlung hängt vom auslösenden Trigger und der Schwere der Erkrankung ab. Der Therapie des Auslösers kommt dabei eine besondere Bedeutung zu.

\section{》) Eine auslösende Virusinfek- tion sollte möglichst behandelt werden}

Liegt eine Virusinfektion als Auslöser vor, sollte diese - sofern möglich - behandelt werden. Bei der Epstein-BarrVirus(EBV)-assoziierten HLH ist die Gabe von Rituximab in vielen Fällen sinnvoll. Die Therapie bakterieller und parasitärer Erkrankungen sowie von Pilzinfektionen kann durch Elimination des Triggers zur Remission der HLH führen. Zudem scheint der Einsatz von Immunglobulinen bei Patienten mit infektiös getriggerter HLH zu einer Verbesserung der Prognose zu führen [14].

Bei Patienten, bei denen die alleinige Gabe von Kortikosteroiden das inflam- matorische Geschehen nicht ausreichend unterdrückt, ist die zusätzliche Gabe von Etoposid zu erwägen, sofern nicht ein zwischenzeitlich detektierter Trigger eine spezifische Therapie vorgibt. Die Kombination aus Dexamethason und Etoposid wurde bei Kindern mit HLH gut untersucht und stellt in modifizierter Form mit einer niedrigeren Etoposiddosis auch beim erwachsenen Patienten eine wirksame Therapieoption dar $[9,10]$. Es ist generell erwähnenswert, dass einige Infektionen wegen des autoaggressiven inflammatorischen Geschehens antiintuitiv zunächst vorrangig immunsuppressiv behandelt werden müssen, um irreversible Organschäden zu verhindern.

\section{》) Malignomassoziierte $\mathrm{HLH}$ treten am häufigsten bei Patienten mit hämatologischen Neoplasien auf}

Malignomassoziierte HLH treten am häufigsten bei Patienten mit hämatologischen Neoplasien auf. Vor allem verschiedene Lymphomsubtypen scheinen gehäuft mit einer HLH assoziiert zu sein. Eine HLH im Zusammenhang mit einem soliden Tumor ist nur selten anzutreffen [2, 20, 25]. Die Therapie der malignomassoziierten HLH stellt eine Herausforderung dar, da der Allgemeinzustand bei einem relevanten Anteil der Patienten die eigentlich indizierte chemotherapeutische Behandlung des HLH-auslösenden Malignoms nicht zulässt. Hier kann eine Vorphasetherapie mit Kortikosteroiden und Etoposid mit dem Ziel des Erhalts und der Verbesserung der Organfunktionen der malignomspezifischen Therapie vorgeschaltet werden. Es muss auch beachtet werden, dass bei Vorliegen einer HLHassoziierten Neutropenie mit dem Beginn der Chemotherapie im Gegensatz zur chemotherapieinduzierten Neutropenie nicht zugewartet werden sollte, da sie Ausdruck des HLH-bedingten inhibitorischen Zytokinsturms und nicht toxisch vermittelt ist. Von der malignomgetriggerten $\mathrm{HLH}$ ist die HLH nach Chemotherapie zu unterscheiden, die in der Regel durch Infektionen aus- 
gelöst wird. Hier sollten Kortikosteroide und gegebenenfalls Immunglobuline verabreicht und eine $\mathrm{zu}$ rasche Fortsetzung der Chemotherapie vermieden werden [20].

Patienten mit autoimmunologischen bzw. rheumatologischen Grunderkrankungen als Auslöser stellen die dritte große Gruppe von Patienten mit HLH auf der Intensivstation dar. Patienten mit MAS-HLH erhalten zunächst hoch dosiert Kortikosteroide, im weiteren Verlauf je nach Triggererkrankung zytokingerichtete Biologika. So gibt es beim MAS-HLH zunehmend Evidenz für die Wirksamkeit des IL-1-Rezeptor-Antagonisten Anakinra [29]. Die hierbei verwendeten Dosen liegen oberhalb des Zulassungsbereichs in anderen Indikationen.

\section{》) Beim MAS-HLH existiert Evidenz für die Wirksamkeit des IL-1-Rezeptor-Antagonisten Anakinra}

Auch die Prognose der terminologisch und pathophysiologisch unscharf definierten MAS-ähnlichen Sepsis, die durch das Vorliegen einer hepatobiliären Dysfunktion und einer disseminierten intravasalen Gerinnung gekennzeichnet ist, kann der Subgruppenanalyse einer randomisierten Sepsisstudie zufolge durch die Gabe von Anakinra verbessert werden [24].

In jüngerer Vergangenheit wurde das Auftreten einer HLH-ähnlichen Symptomatik auch bei kritisch an „coronavirus disease 2019“ (COVID-19) erkrankten Patienten beobachtet. Hier legen präliminäre Daten jedoch nahe, dass trotz des Nachweises einer Hyperinflammation klinisch und laborchemisch für die HLH charakteristische Befunde sehr selten anzutreffen sind und gemäß HScore nur wenige Patienten eine hohe Wahrscheinlichkeit für das Vorliegen einer HLH haben. Dennoch konnte für Patienten mit schwerer COVID-19-Erkrankung und starker Entzündungsreaktion gezeigt werden, dass eine immunsuppressive Therapie den Verlauf günstig beeinflusst $[11,17]$. Daher wird bei Patienten mit

Wien klin Mag 2021 · 24:246-251 https://doi.org/10.1007/s00740-021-00414-z (c) Springer-Verlag GmbH Austria, ein Teil von Springer Nature 2021

\section{A. Eichenauer · G. Lachmann · P. La Rosée \\ Die hämophagozytische Lymphohistiozytose bei kritisch kranken Patienten}

\section{Zusammenfassung}

Bei der hämophagozytischen Lymphohistiozytose (HLH) handelt es sich um ein Hyperinflammationssyndrom bedingt durch aberrant aktivierte Makrophagen und T-Zellen. Beim Erwachsenen ist in erster Linie die erworbene Form anzutreffen. Häufige Auslöser sind Infektionen, Malignome und Autoimmunerkrankungen. Zuletzt wurden zudem zunehmend Fälle berichtet, in denen das Auftreten im Zusammenhang mit stattgehabten Immuntherapien zu sehen war. Auf der Intensivstation ist die HLH aufgrund des ähnlichen klinischen Erscheinungsbilds oft schwer von der Sepsis abzugrenzen. Zum Teil liegen beide zeitgleich vor. Die frühzeitige Diagnosestellung und Einleitung einer adäquaten immunsuppressiven Therapie ist für den weiteren Verlauf und die Prognose der HLH essenziell. Deshalb muss bei kritisch kranken Patienten mit persistierendem Fieber und entsprechenden Symptomen (z.B. Splenomegalie, neurologische Auffäl- ligkeiten) oder Laborveränderungen (z. B. erhöhter Ferritinwert, Zytopenie von 2 oder 3 Zellreihen, erhöhte Transaminasen) das Vorliegen einer HLH in Betracht gezogen werden. Die Diagnose wird mithilfe der HLH2004-Kriterien gestellt. Mit dem HScore kann die Wahrscheinlichkeit des Vorliegens einer HLH berechnet werden. Hochdosierte Kortikosteroide stellen den Grundpfeiler der HLH-Therapie dar. Je nach Auslöser werden Etoposid, Immunglobuline, Anakinra oder weitere Medikamente ergänzt. Der Verlauf hängt neben einem frühzeitigen Behandlungsbeginn vom Auslöser sowie dem Ansprechen auf die Therapie ab. Insgesamt ist die Prognose der HLH trotz maximaler intensivmedizinischer Behandlung ungünstig und sie ist mit einer hohen Letalität assoziiert.

Schlüsselwörter

Intensivstation · Diagnostische Techniken und Verfahren · Therapie · Prognose

\section{Hemophagocytic lymphohistiocytosis in critically ill patients}

\section{Abstract}

Hemophagocytic lymphohistiocytosis (HLH) is a hyperinflammation syndrome. In adults, secondary $\mathrm{HLH}$ is mostly observed. $\mathrm{HLH}$ is often triggered by infections, malignancies or autoimmune disorders. However, HLH cases in association with immunotherapies have been described recently. HLH in critically ill patients is often difficult to differentiate from sepsis. Both conditions can also be present at the same time. Early diagnosis and timely initiation of an adequate immunosuppressive therapy are essential for the further course and the prognosis of $\mathrm{HLH}$. Therefore, $\mathrm{HLH}$ should represent a differential diagnosis in critically ill patients with persistent fever and additional symptoms (e.g. enlarged spleen, neurologic symptoms) or laboratory parameters (e.g. hyperferritinemia, cytopenia, increased transaminases) compatible with $\mathrm{HLH}$. The diagnosis of $\mathrm{HLH}$ is made using the HLH-2004 criteria. The probability of the presence of $\mathrm{HLH}$ can be calculated using the HScore. High-dose corticosteroids represent the cornerstone of HLH treatment. Etoposide, immunoglobulins, anakinra or other drugs are added depending on the trigger. The course of HLH is influenced by the time of treatment initiation, the underlying trigger and the response to treatment. Generally, the prognosis of critically ill HLH patients is poor.

\section{Keywords}

Intensive care unit - Diagnostic techniques and procedures - Therapy · Prognosis
COVID-19-Erkrankung, die Sauerstoff benötigen oder invasiv beatmet werden, die Gabe von Dexamethason empfohlen.

Ebenfalls wurde in den letzten Jahren vermehrt über ein hyperinflammatorisches Geschehen im Zusammenhang mit zellulären und antikörperbasierten Immuntherapien berichtet. Es wird je- doch nur selten das Stadium der HLH erreicht. Je nach Auslöser wird mit Kortikosteroiden und dem gegen IL-6 gerichteten Antikörper Tocilizumab behandelt [6]. In schweren Fällen ist die Gabe von Etoposid zu diskutieren.

Trotz maximaler intensivmedizinischer Behandlung ist die Prognose von 


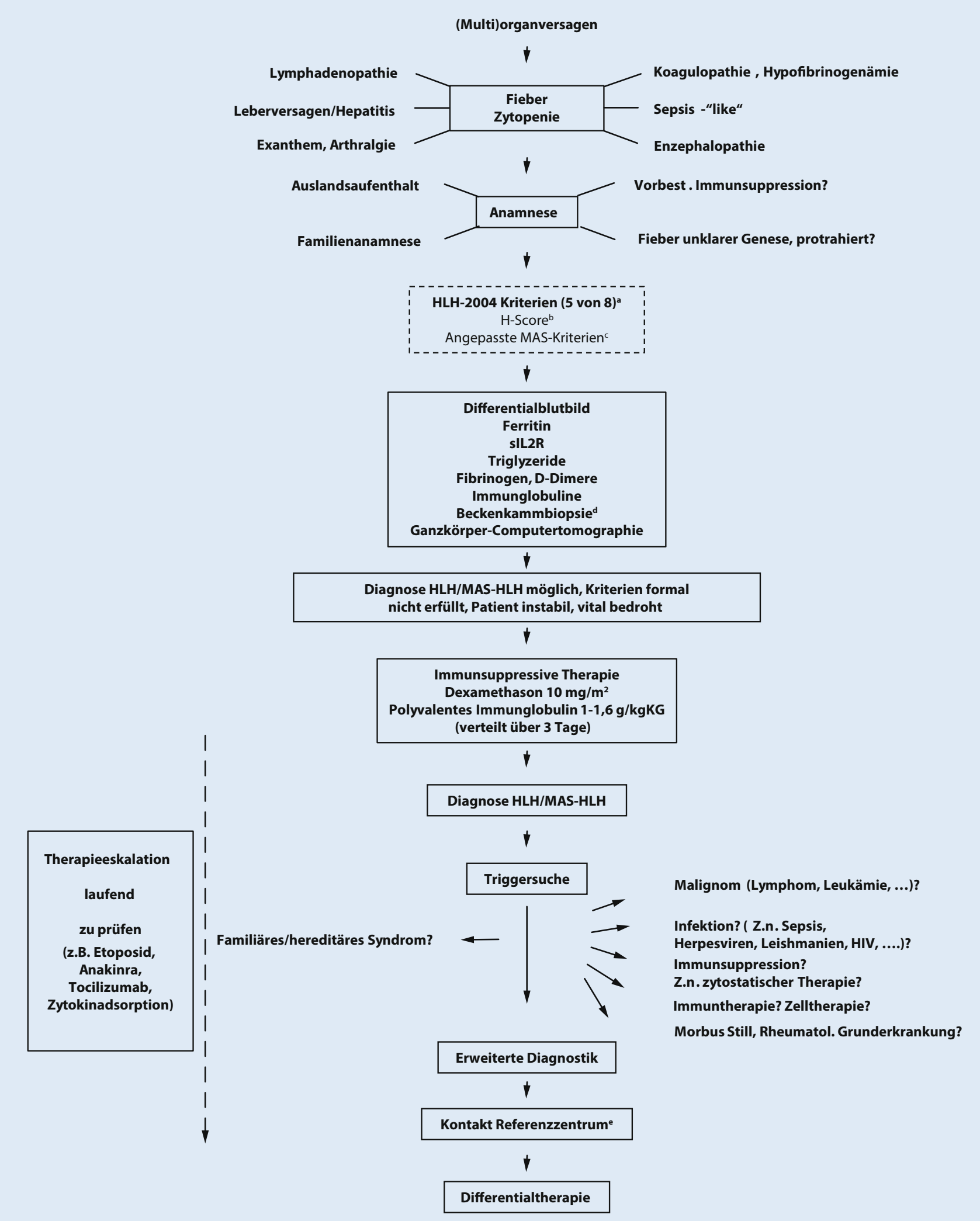

Abb. 1 A Flussdiagramm kritisch kranker Patienten mit HLH/MAS-HLH und (Multi-)Organversagen. ${ }^{a}$ siehe $\bullet$ Tab. 1; ; siehe online Kalkulator unter http://saintantoine.aphp.fr/score/; ${ }^{\mathrm{C}}$ Quelle [23]; ${ }^{\mathrm{d}}$ Zytologie, Durchflusszytometrie, Histologie, Infektiologie (z. B. Polymerasekettenreaktion bezüglich Tuberkulose, Leishmanien); ${ }^{e}$ www.hlh-registry.org. HLH Hämophagozytische Lymphohistiozytose, MAS-HLH Makrophagenaktivierungssyndrom, sIL2R löslicher Interleukin-2-Rezeptor 
kritisch kranken Patienten mit HLH schlecht. So lag die Mortalität unter HLH-Patienten, die auf einer Intensivstation behandelt wurden, gemäß einer kürzlich publizierten systematischen Übersichtsarbeit bei knapp $60 \%$, unterschied sich jedoch zwischen verschiedenen Patientengruppen in Abhängigkeit vom auslösenden Trigger. Am ungünstigsten war die Prognose bei $\mathrm{Pa}$ tienten mit malignomassoziierter $\mathrm{HLH}$ [14].

\section{Weitere Therapieoptionen}

Insgesamt 63 HLH-Patienten mit unzureichendem Ansprechen auf die Erstlinientherapie bestehend aus Kortikosteroiden und Etoposid wurden im Rahmen einer prospektiven Studie mit dem DEPSchema (liposomales Doxorubicin, Etoposid, Methylprednisolon) behandelt. Die Ansprechrate lag bei 76,2\% [26]. Bei 26 pädiatrischen Patienten mit refraktärer EBV-assoziierter HLH wurde zudem das DEP-Schema in Kombination mit pegylierter (PEG-)Asparaginase untersucht. Hier lag die Ansprechrate bei $61,5 \%$ [28].

Eine weitere Therapieoption stellt der Januskinase(JAK)-2-Inhibitor Ruxolitinib dar, zu dessen Einsatz bei der HLH ebenfalls prospektive Daten vorliegen. Bei 12 Kindern mit in der Mehrzahl der Fälle EBV-assoziierter HLH, die über 28 Tage Ruxolitinib erhalten hatten, wurde eine Ansprechrate von 83,3\% beobachtet. Die Rate an Patienten mit ereignisfreiem Überleben nach 6 Monaten lag bei 58,8\% [27]. Auch bei 5 erwachsenen Patienten mit sekundärer $\mathrm{HLH}$, die im Rahmen einer Pilotstudie mit Ruxolitinib behandelt wurden, konnte eine klinische und laborchemische Verbesserung der HLH-assoziierten Parameter nachgewiesen werden [1].

》) Der JAK2-Inhibitor Ruxolitinib stellt eine weitere Therapieoption dar

Eine laufende Studie untersucht bei Patienten mit sekundärer HLH den Interferon- $\gamma$-Antikörper Emapalumab (NCT03985423). Zuvor war der Anti- körper bereits prospektiv bei Kindern mit primärer HLH getestet worden. Dort war es bei mehr als der Hälfte der Patienten zu einem Ansprechen gekommen [21]. Einzelfallberichte zum Einsatz von Emapalumab liegen auch für erwachsene Patienten mit MAS-HLH bereits vor [7].

Bei Patienten im Multiorganversagen kann in Einzelfällen eine Zytokinelimination mittels Plasmapherese oder Adsorptionssäule durchgeführt werden, um insbesondere bei Leber- und Nierenversagen die Zeit bis zur möglichen Pharmakotherapie zu überbrücken [8].

\section{Fazit für die Praxis}

- Die hämophagozytische Lymphohistiozytose (HLH) ist bei kritisch kranken Patienten auf der Intensivstation aufgrund der unspezifischen Symptomatik unterdiagnostiziert.

- Das Vorliegen einer HLH sollte differenzialdiagnostisch bei Patienten mit protrahiertem Fieber, Zytopenien und Vergrößerung von Milz und/ oder Leber in Betracht gezogen werden, wenn das Ansprechen auf eine antiinfektive Therapie ausbleibt.

- Laborchemisch untermauern vor allem Extremwerte für Ferritin die Verdachtsdiagnose HLH.

- Der Grundpfeiler der HLH-Behandlung besteht in einer immunsuppressiven Therapie mit hochdosierten Kortikosteroiden.

- Neben der medikamentösen Immunsuppression muss - sofern identifiziert - die Therapie des Triggers erfolgen.

- Trotz immunsuppressiver Therapie ist die Letalität bei kritisch kranken HLHPatienten noch immer sehr hoch.

\section{Korrespondenzadresse}

Dr. Dennis A. Eichenauer

Klinik I für Innere Medizin, Zentrum für Integrierte Onkologie Aachen Bonn Köln Düsseldorf, Uniklinik Köln Kerpener Str. 62, 50937 Köln, Deutschland dennis.eichenauer@uk-koeln.de
Einhaltung ethischer Richtlinien

Interessenkonflikt. D. A. Eichenauer, G. Lachmann und P. La Rosée geben an, dass kein Interessenkonflikt besteht.

Für diesen Beitrag wurden von den Autoren keine Studien an Menschen oder Tieren durchgeführt. Für die aufgeführten Studien gelten die jeweils dort angegebenen ethischen Richtlinien.

\section{Literatur}

1. Ahmed A, Merrill SA, Alsawah F et al (2019) Ruxolitinib in adult patients with secondary haemophagocytic lymphohistiocytosis: an openlabel, single-centre, pilot trial. Lancet Haematol 6:e630-e637. https://doi.org/10.1016/\$23523026(19)30156-5

2. Birndt S, Schenk T, Heinevetter B et al (2020) Hemophagocytic lymphohistiocytosis in adults: collaborative analysis of 137 cases of a nationwide German registry. J Cancer Res Clin Oncol 146:1065-1077. https://doi.org/10.1007/s00432020-03139-4

3. Brisse E, Wouters CH, Matthys P (2016) Advances in the pathogenesis of primary and secondary haemophagocytic lymphohistiocytosis: differences and similarities. Br J Haematol 174:203-217. https://doi.org/10.1111/bjh.14147

4. Debaugnies F, Mahadeb B, Ferster A et al (2016) Performances of the $\mathrm{H}$-score for diagnosis of hemophagocytic lymphohistiocytosis in adult and pediatric patients. Am J Clin Pathol 145:862-870. https://doi.org/10.1093/ajcp/aqw076

5. Fardet L, Galicier L, Lambotte $O$ et al (2014) Development and validation of the HScore, a score for the diagnosis of reactive hemophagocytic syndrome. Arthritis Rheumatol 66:2613-2620. https://doi.org/10.1002/art.38690

6. Fishman JA, Hogan JI, Maus MV (2019) Inflammatory and infectious syndromes associated with cancer immunotherapies. Clin Infect Dis 69:909-920. https://doi.org/10.1093/cid/ciy1025

7. Gabr JB, Liu E, Mian S et al (2020) Successful treatment of secondary macrophage activation syndrome with emapalumab in a patient with newly diagnosed adult-onset Still's disease: case report and review of the literature. Ann TransI Med 8:887.https://doi.org/10.21037/atm-20-3127

8. Greil C, Roether F, La Rosée P et al (2017) Rescue of cytokine storm due to HLH by hemoadsorption in a CTLA4-deficient patient. J Clin Immunol 37:273-276. https://doi.org/10.1007/s10875017-0377-7

9. Henter Jl, Chow CB, Leung CW, Lau YL (2006) Cytotoxic therapy for severe avian influenza $A$ (H5N1) infection. Lancet 367:870-873. https://doi. org/10.1016/S0140-6736(06)68232-9

10. Henter J, Horne A, Arico M et al (2007) HLH2004: Diagnostic and therapeutic guidelines for hemophagocytic lymphohistiocytosis. Pediatr Blood Cancer 48:124-131. https://doi.org/10. 1002/pbc.21039

11. Horby P, Lim WS, Emberson JR et al (2020) Dexamethasone in hospitalized patients with Covid-19-preliminary report. N Engl J Med. https://doi.org/10.1056/NEJMoa2021436

12. Kapoor S, Morgan CK, Siddique MA, Guntupalli KK (2018) Intensive care unit complications and outcomes of adult patients with hemophagocytic 
lymphohistiocytosis: a retrospective study of 16 cases. World J Crit Care Med 7:73-83. https:// doi.org/10.5492/wjccm.v7.i6.73

13. Knaak C, Nyvlt P, Schuster FS et al (2020) Hemophagocytic lymphohistiocytosis in critically ill patients: diagnostic reliability of $\mathrm{HLH}-2004$ criteria and HScore. Crit Care 24:244. https://doi. org/10.1186/s13054-020-02941-3

14. KnaakC, Schuster FS, Nyvlt Petal (2020) Treatment and mortality of hemophagocytic lymphohistiocytosis in adult critically ill patients: a systematic review with pooled analysis. Crit Care Med. https:// doi.org/10.1097/CCM.0000000000004581

15. Knaak C, Schuster FS, Spies C et al (2020) Hemophagocytic lymphohistiocytosis in critically ill patients. Shock 53:701-709. https://doi.org/10. 1097/SHK.0000000000001454

16. La Rosée $P$, Horne $A$, Hines $M$ et al (2019) Recommendations for the management of hemophagocytic lymphohistiocytosis in adults. Blood 133:2465-2477. https://doi.org/10.1182/ blood.2018894618

17. La Rosée $P$, Bremer $H C$, La Rosée $F$ et al (2020) Interdisciplinary COVID board for patients with SARS-CoV-2-triggered hyperferritinemic inflammation. Med Klin Intensivmed Notfmed 28:20-750

18. Lachmann G, Spies C, Schenk T et al (2018) Hemophagocytic lymphohistiocytosis: potentially underdiagnosed in intensive care units. Shock 50:149-155. https://doi.org/10.1097/SHK. 0000000000001048
19. Lachmann G, Knaak C, Vorderwulbecke $G$ et al (2020) Hyperferritinemia in critically ill patients. Crit Care Med 48:459-465. https://doi.org/10. 1097/CCM.0000000000004131

20. Lehmberg K, Sprekels B, Nichols KE et al (2015) Malignancy-associated haemophagocytic lymphohistiocytosis in children and adolescents. $\mathrm{Br} J$ Haematol 170:539-549. https://doi.org/10.1111/ bjh.13462

21. Locatelli F, Jordan MB, Allen C et al (2020) Emapalumab in children with primary hemophagocytic lymphohistiocytosis. N Engl J Med 382:1811-1822. https://doi.org/10.1056/NEJMoa1911326

22. Meena NK, Sinokrot O, Duggal A et al (2019) The performance of diagnostic criteria for hemophagocytic lymphohistiocytosis in critically ill patients. J Intensive Care Med. https://doi.org/ $10.1177 / 0885066619837139$

23. Ravelli A et al (2016) 2016 classification criteria for macrophage activation syndrome complicating systemic juvenile idiopathic arthritis: a European league against rheumatism/American college of rheumatology/paediatric rheumatology international trials organisation collaborative initiative. Ann Rheum Dis 75:481-489

24. Shakoory B, Carcillo JA, Chatham WW et al (2016) Interleukin-1 receptor blockade is associated with reduced mortality in sepsis patients with features of macrophage activation syndrome: reanalysis of a prior phase III trial. Crit Care Med 44:275-281. https://doi.org/10.1097/CCM.0000000000001402

25. Wang H, Xiong L, Tang W et al (2017) A systematic review of malignancy-associated hemophagocytic lymphohistiocytosis that needs more attentions. Oncotarget 8:59977-59985. https://doi.org/10. 18632/oncotarget.19230

26. Wang Y, Huang W, Hu L et al (2015) Multicenter study of combination DEP regimen as a salvage therapy for adult refractory hemophagocytic lymphohistiocytosis. Blood 126:2186-2192. https:// doi.org/10.1182/blood-2015-05-644914

27. Zhang $\mathrm{Q}$, Wei $\mathrm{A}, \mathrm{Ma} \mathrm{H}-\mathrm{H}$ et al (2020) A pilot study of ruxolitinib as a front-line therapy for 12 children with secondary hemophagocytic lymphohistiocytosis. Haematologica. https://doi. org/10.3324/haematol.2020.253781

28. Zhao Y, Li Z, Zhang L et al (2020) L-DEP regimen salvage therapy for paediatric patients with refractory Epstein-Barr virus-associated haemophagocytic lymphohistiocytosis. Br Haematol. https://doi.org/10.1111/bjh.16861

29. Zhou S, Qiao J, Bai J et al (2018) Biological therapy of traditional therapy-resistant adult-onset still's disease: an evidence-based review. Ther Clin Risk Manag 14:167-171. https://doi.org/10.2147/ TCRM.S155488

Hier steht eine Anzeige.

\section{黑 Springer}

